\title{
THE 2012 RISE OF THE REMARKABLE TYPE IIn SN 2009ip
}

\author{
José L. Prieto ${ }^{1}$, J. Brimacombe 2,3 , A. J. Drake ${ }^{4}$, and S. Howerton ${ }^{5}$ \\ ${ }^{1}$ Department of Astrophysical Sciences, Princeton University, NJ 08544, USA \\ 2 James Cook University, Cairns Base Hospital, Cairns, Australia \\ ${ }^{3}$ Coral Towers Observatory, Unit 38 Coral Towers, 255 Esplanade, Cairns 4870, Australia \\ ${ }^{4}$ California Institute of Technology, 1200 E. California Blvd., CA 91225, USA \\ 51401 South A, Arkansas City, KS 67005, USA \\ Received 2012 October 11; accepted 2012 December 20; published 2013 January 11
}

\begin{abstract}
Recent observations by Mauerhan et al. have shown the unprecedented transition of the previously identified luminous blue variable (LBV) and supernova (SN) impostor SN 2009ip to a real Type IIn SN explosion. We present $\sim 100$ optical $R$ - and $I$-band photometric measurements of SN 2009ip obtained between UT 2012 September 23.6 and October 9.6, using 0.3-0.4 m aperture telescopes from the Coral Towers Observatory in Cairns, Australia. The light curves show well-defined phases, including very rapid brightening early on $(0.5$ mag in $6 \mathrm{hr}$ observed during the night of September 24), a transition to a much slower rise between September 25 and September 28, and a plateau/peak around October 7. These changes are coincident with the reported spectroscopic changes that most likely mark the start of a strong interaction between the fast SN ejecta and a dense circumstellar medium formed during the LBV eruptions observed in recent years. In the 16-day observing period, SN 2009ip brightened by $3.7 \mathrm{mag}$ from $I=17.4 \mathrm{mag}$ on September $23.6\left(M_{I} \simeq-14.2\right)$ to $I=13.7 \mathrm{mag}\left(M_{I} \simeq-17.9\right)$ on October 9.6, radiating $\sim 3 \times 10^{49} \mathrm{erg}$ in the optical wavelength range. As of 2012 October 9.6, SN 2009ip is more luminous than most Type IIP SN and comparable to other Type IIn SN.
\end{abstract}

Key words: circumstellar matter - stars: evolution - stars: mass-loss - supernovae: individual (SN 2009ip)

Online-only material: color figures, machine-readable table

\section{INTRODUCTION}

Establishing an observational mapping between the properties of core-collapse supernova (SN) explosions (and related luminous outbursts) and local populations of massive stars is key for constraining stellar evolution theory (e.g., Langer 2012). Arguably, the best link so far comes from the direct detections of red supergiants with main-sequence masses $M \simeq 8-15 M_{\odot}$ as progenitors of Type IIP supernovae (e.g., Smartt 2009 and references therein), the most common kind of core-collapse SN. However, direct detections of SN progenitors with higher masses $\left(M \gtrsim 20 M_{\odot}\right)$ have been elusive (e.g., Kochanek et al. 2008; Smartt 2009).

The detection of a very luminous star $\left(M_{V} \sim-10 \mathrm{mag}\right)$ identified in pre-explosion images at the location of the Type IIn 6 SN $2005 \mathrm{gl}$ provided the first direct evidence for a very massive H-rich star $\left(\gtrsim 50 M_{\odot}\right)$ that exploded as a luminous core-collapse SN (Gal-Yam et al. 2007; Gal-Yam \& Leonard 2009). These observations lend support for the connection made initially by Smith et al. (2007) between one of the most luminous Type IIn SN 2006gy and very massive $\left(\sim 100 M_{\odot}\right)$ luminous blue variable (LBV) stars like $\eta$ Carinae. There is now mounting evidence of this connection (e.g., SN 2006jc, Pastorello et al. 2007; SN 2010jl, Smith et al. 2011a; SN 1961V, Kochanek et al. 2011; Smith et al. 2011b), which challenges standard massive stellar evolution theory (but, see, e.g., Woosley et al. 2007; Arnett \& Meakin 2011; Quataert \& Shiode 2012; Chevalier 2012).

Luckily, in 2012 September nature has been kind and provided the best case to date of a very massive star exploding as a bright core-collapse SN: SN 2009ip. This transient was discovered on 2009 August 29 by the CHASE survey in the

\footnotetext{
6 Dominated by narrow $\mathrm{H}$ lines in emission, which indicates a strong
} interaction between the fast ejecta and a dense circumstellar medium. outskirts of the galaxy NGC 7259 (Maza et al. 2009) and was initially given an SN name by the IAU. However, detailed photometric and spectroscopic studies by Smith et al. (2010) and Foley et al. (2011) showed that it never quite reached SN status. It was actually an $\mathrm{SN}$ impostor ${ }^{7}$ (e.g., Humphreys \& Davidson 1994; Kochanek et al. 2012), an LBV with estimated mass $M \simeq 50-80 M_{\odot}$ that had an eruptive mass-loss event. The Catalina Real-Time Transient Survey discovered another outburst of comparable amplitude during 2010 (Drake et al. 2010) and a third outburst on 2012 July 24 (Drake et al. 2012), the brightest so far. The initial optical spectra obtained by Foley et al. (2012) on August 26 showed narrow emission lines of $\mathrm{H}$ and $\mathrm{He} \mathrm{I}$, consistent with the spectra obtained in the 2009 outburst. However, an unprecedented change of the spectrum was reported by Smith \& Mauerhan (2012a). On September 15 and 16 they detected very broad emission lines with P-Cygni profiles, consistent with features observed in real Type II SN; the massive LBV star had probably exploded as an SN in real time! Another dramatic change in the spectral properties was reported by Smith \& Mauerhan (2012b), by September 28 the broad P-Cygni features had mostly disappeared, leaving behind narrow features characteristic of Type IIn SN. All the details of their discovery and their follow-up observations can be found in Mauerhan et al. (2012a, hereafter M12a). Pastorello et al. (2012, hereafter P12) have also presented detailed follow-up observations of SN 2009ip and propose that the latest outburst might not be the final core-collapse explosion, but another eruption within the pulsational pair-instability mechanism of Woosley et al. (2007).

In this Letter, we present optical observations of SN 2009ip that clearly resolve in time the brightening initially reported by

\footnotetext{
7 With $M \sim-14$, similar to $\eta$ Carinae's Great Eruption, but significantly shorter timescale.
} 


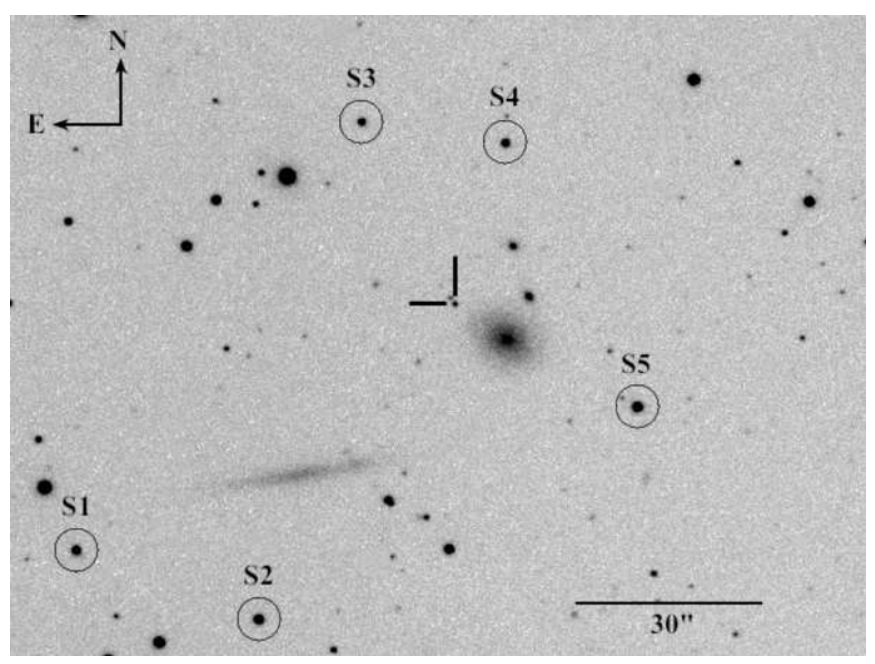

Figure 1. Finding chart of SN 2009ip. This image was obtained with the IR luminance filter $(>700 \mathrm{~nm}$ ) on 2012 September 24 . The circles mark the local standard stars used to estimate the photometric zero points and the tick marks show the position of SN 2009ip.

Brimacombe (2012). In Section 2 we discuss the observations, data reduction, and extraction of optical light curves using difference imaging photometry. In Section 3 we present an analysis of the different phases of the light curve. In Section 4 we present a discussion of the observed properties. In Section 5 we present the conclusions of this work. We adopt a distance of $20.4 \mathrm{Mpc}(\mu=31.55 \mathrm{mag})$ and Galactic extinction of $A_{V}=0.06$ mag toward SN 2009ip (M12a) throughout the Letter. All the dates presented in this Letter are UT.

\section{OBSERVATIONS AND PHOTOMETRY}

Imaging of the field of SN 2009ip was obtained by one of us (J.B.) between 2012 September 23.6 and October 9.6 from the Coral Towers Observatory (Cairns, Australia). The data were collected from two different telescopes using different broadband filters: $33 \mathrm{~cm}$ RCOS (with an $R$ filter) and $41 \mathrm{~cm}$ RCOS (with an IR luminance filter, which has sharp blue cutoff at $700 \mathrm{~nm}^{8}$ ). The CCD cameras used in both telescopes are identical $3 \mathrm{k} \times 2 \mathrm{k}$ SBIG STL6K, and give a total field of view of $25^{\prime} \times 17^{\prime}\left(1^{\prime \prime}\right.$ pixel $^{-1}$ image scale binned $\left.2 \times 2\right)$. All the images were obtained using an exposure time of $900 \mathrm{~s}$. A section of an IR image of SN 2009ip obtained on September 24 is shown in Figure 1.

We used the software MaxIm DL (version 4.62) to complete the initial data reduction, which consists of bias subtraction, dark subtraction, and flat fielding. After visually inspecting all images and rejecting frames affected by bad tracking, we kept $85(R)$ and 118 (IR) frames for further photometric analysis. We used the image subtraction package ISIS2 (Alard 2000) to extract the fluxes of SN 2009ip in individual frames following the steps described in Hartman et al. (2004). As reference images, we used stacks of 5-10 images with good seeing obtained on September 24-25. We carried out point-spread function photometry with the DAOPHOT II package (Stetson 1992) to subtract the SN flux from the best-seeing images and used the resulting $\mathrm{SN}$-subtracted images as the final reference frames.

The SN fluxes obtained with ISIS2 in the $R$ and $I$ images were calibrated to standard magnitudes using data from the

\footnotetext{
8 The total throughput of this system (filter + CCD + atmosphere) is $\sim 30 \%$ broader than the Bessell $I$ band with $\lambda_{\text {eff }}=835.8 \mathrm{~nm}$ and $\mathrm{FWHM}=200.6 \mathrm{~nm}$.
}

Table 1

Light Curve of SN 2009ip

\begin{tabular}{lcccc}
\hline \hline UT Date & HJD $-2,456,000$ & mag & $\sigma$ & Filter \\
\hline Sep 24.536400 & 195.040909 & 16.549 & 0.047 & $R$ \\
Sep 24.552130 & 195.056641 & 16.494 & 0.045 & $R$ \\
Sep 24.567801 & 195.072327 & 16.382 & 0.040 & $R$ \\
Sep 24.583472 & 195.087997 & 16.403 & 0.043 & $R$ \\
Sep 24.599144 & 195.103668 & 16.356 & 0.044 & $R$ \\
Sep 24.614826 & 195.119370 & 16.256 & 0.039 & $R$ \\
Sep 24.633634 & 195.138153 & 16.211 & 0.040 & $R$ \\
Sep 24.644965 & 195.149475 & 16.259 & 0.043 & $R$ \\
Sep 24.656285 & 195.160797 & 16.256 & 0.040 & $R$ \\
Sep 24.667593 & 195.172134 & 16.250 & 0.038 & $R$ \\
$\ldots$ & $\ldots$ & $\ldots$ & $\ldots$ & $R$ \\
\hline
\end{tabular}

(This table is available in its entirety in a machine-readable form in the online journal. A portion is shown here for guidance regarding its form and content.)

AAVSO Photometric All-Sky Survey (APASS ${ }^{9}$ ). We obtained from APASS calibrated Sloan $r^{\prime}$ and $i^{\prime}$ magnitudes of five isolated stars in the field of SN 2009ip. We converted these magnitudes in the SDSS photometric system to standard $R I$ (Johnson/Kron-Cousins) photometry using the transformation equations obtained by R. Lupton. ${ }^{10}$ In order to estimate the zero points, we performed aperture photometry of the five local standard stars in the reference images using a 9" radius aperture with the IRAF task phot. The resulting average zero points in the $R I$ filters have a standard deviation of 0.02 mag. The final photometry of SN 2009ip calibrated using these zero points is presented in Table 1 and the light curves are shown in Figure 1. The errors in each magnitude estimate include Poisson errors from ISIS2 as well as the estimated standard error in the photometric zero points. Our photometry is in good agreement with the results presented in $\mathrm{P} 12$, with mean differences during the period of observations of $-0.01 \pm 0.01 \mathrm{mag}$ in $R$ and $-0.02 \pm 0.03 \mathrm{mag}$ in $I$.

\section{ANALYSIS}

The high cadence and number of observations allow us to clearly resolve in time the brightening of SN 2009ip, initially reported in Brimacombe (2012) and Prieto et al. (2012). The optical light curves presented in Figure 2 show well-defined phases: (1) approximately constant magnitude (September 23); (2) very rapid brightening in a period of hours (September 24-25); (3) turnover to significantly slower brightening (September 25-28); and (4) slow brightening, reaching peak magnitude (September 30-October 9). We describe these phases in more detail below.

Between September 23.56 and 23.66, the magnitude of SN 2009ip is consistent with being constant at $I=$ $17.39 \pm 0.05 \mathrm{mag}\left(M_{I} \simeq-14.2\right)$. However, between September 23.66 and 24.45 , SN 2009ip brightened by $\Delta I \simeq 0.65$ mag and continued a very rapid brightening throughout the $6 \mathrm{hr}$ of continuous imaging obtained during the night of September 24. In this period, it brightened between $I=16.7$ (September 24.45) and $I=16.2 \mathrm{mag}$ (September 24.70). A linear fit to the $I$-band rise on September 24 gives a slope of $2.30 \pm 0.10$ mag day $^{-1}$.

Between September 24.70 and 25.38 , the SN brightened by $\Delta I \simeq 1.0 \mathrm{mag}$, reaching $I=15.1 \mathrm{mag}\left(M_{I} \simeq-16.5\right)$. The light

\footnotetext{
9 http://www.aavso.org/apass

10 http://www.sdss.org/dr7/algorithms/sdssUBVRITransform.html \#Lupton2005
} 


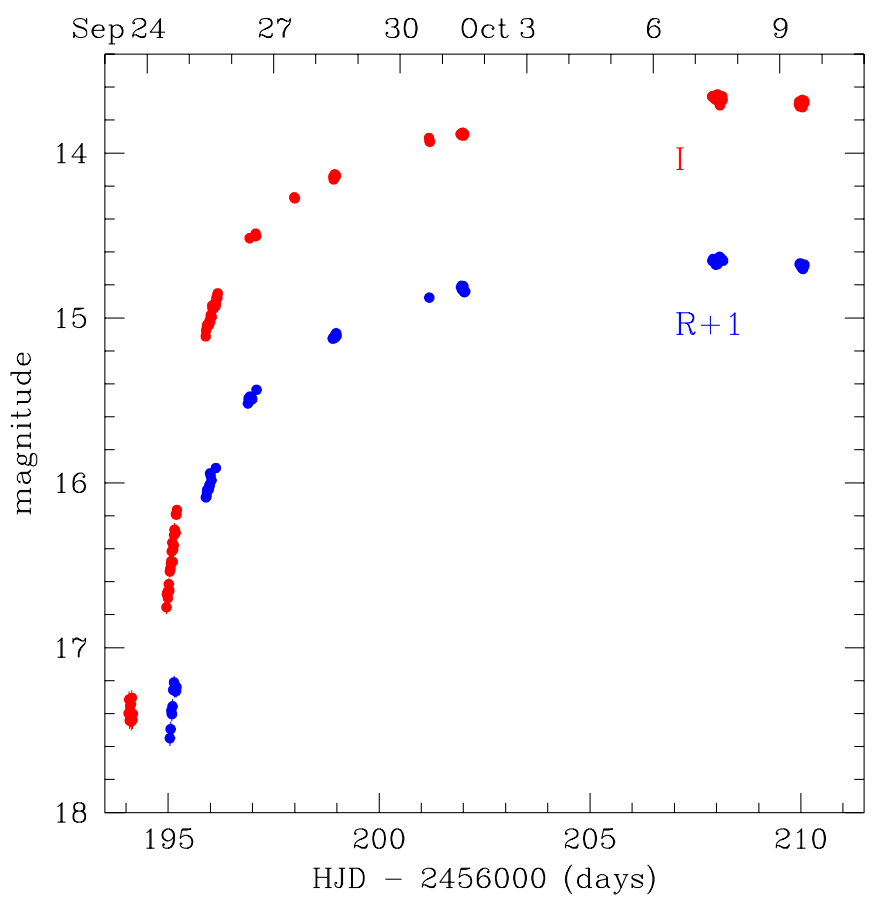

Figure 2. Light curves of SN 2009ip in the $R$ and IR (>700 nm) bands, calibrated to standard $R$ and $I$ magnitudes, respectively. The data were obtained by J. Brimacombe between 2012 September 23.6 and October 9.6 from the Coral Towers Observatory (Cairns, Australia).

(A color version of this figure is available in the online journal.)

curve turned over to a slower brightening on September 25. A linear fit to the data obtained during the night of September 25 gives a slope of $0.79 \pm 0.05 \mathrm{mag} \mathrm{day}^{-1}$. The turnover can be seen clearly in both the $R$ - and $I$-band photometric data obtained between September 25 and September 28 (see Figure 2), reaching $I=14.1 \mathrm{mag}\left(M_{I} \simeq-17.5\right)$ by September 28.47.

The latest part of the light curve between September 30 and October 7 shows a slow and steady brightening at a rate of $0.037 \pm 0.001 \mathrm{mag} \mathrm{day}^{-1}(\mathrm{rms}=0.01 \mathrm{mag})$. However, in our most recent images obtained on October 9.6, the $\mathrm{SN}$ had faded by $\sim 0.03$ mag with respect to the images obtained on October 7.6. The magnitude of SN 2009ip on October 9.6 was $I=13.70 \pm 0.02 \mathrm{mag}\left(M_{I} \simeq-17.9\right)$. The $R-I$ color is consistent with being nearly constant $(R-I=-0.02 \pm 0.03)$ during the period between September 25 and October 9.

\section{DISCUSSION}

Our photometric observations, obtained during a 16-day period between September 23.6 and October 9.6, show that the Type IIn SN 2009ip brightened by $3.7 \mathrm{mag}$ in the optical to an absolute magnitude $M_{R}=M_{I} \simeq-17.9$ (see also Margutti et al. 2012a; M12a; P12) and it reached peak magnitude around October 7 (see Figure 2). Its peak luminosity is in the observed range of Type II SN; in fact it is more luminous than most Type IIP SN (e.g., Li et al. 2011). The comparison to other Type IIn SN is less straightforward since the rates are lower and the samples are incomplete. However, there are several wellstudied Type IIn SN with peak absolute magnitudes around $M \sim-18$ (e.g., Kiewe et al. 2012; Stritzinger et al. 2012; Roming et al. 2012; Mauerhan et al. 2012b).

We can estimate the total radiated energy during the period of the observations presented here. We need an estimate of

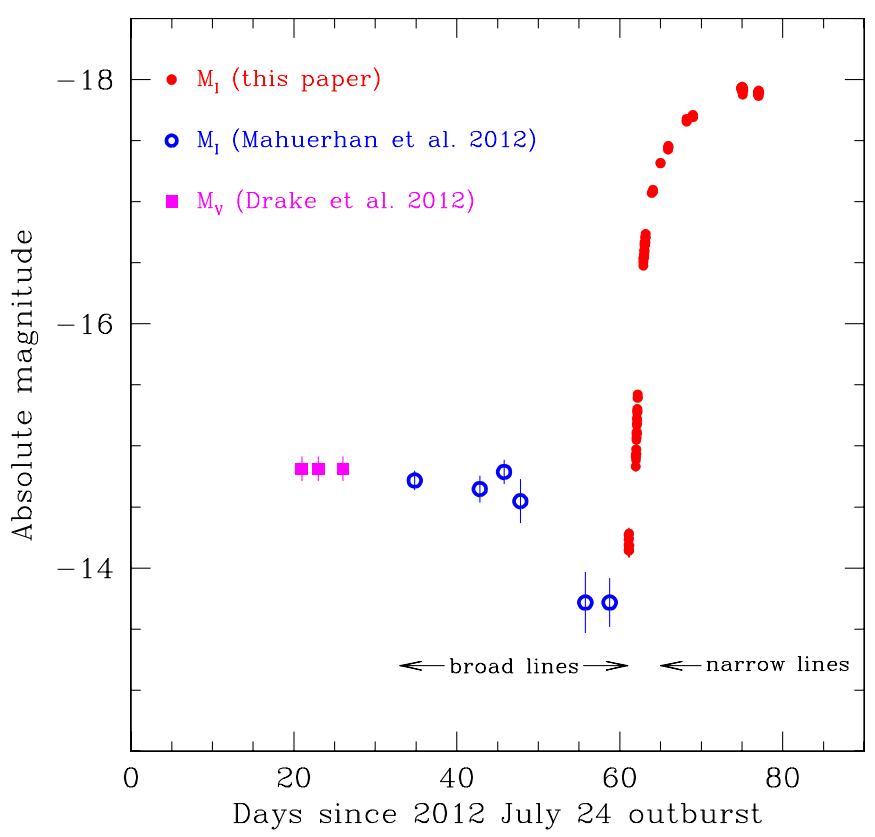

Figure 3. Absolute magnitude light curve of SN 2009ip since its latest eruption was reported on 2012 July 24 by Drake et al. (2012). The filled circles are the $I$-band photometry presented in this Letter; the open circles are I-band photometry reported in Mauerhan et al. (2012a); and the filled squares are $V$-band photometry reported in Drake et al. (2012). The spectrum of SN 2009ip showed broad $\mathrm{H}$ and He I emission lines with P-Cygni profiles $\left(v \simeq 10,000 \mathrm{~km} \mathrm{~s}^{-1}\right)$, characteristic of SN shock speeds, starting on (or before) August 26 (Vinko et al. 2012; but see Foley et al. 2012) until, at least, September 23 (Mauerhan et al. 2012a). However, by September 26-27 the spectrum had changed substantially (Mauerhan et al. 2012a; Burgasser et al. 2012; Vinko et al. 2012) and the emission lines became strong and narrow $\left(v \sim 1000 \mathrm{~km} \mathrm{~s}^{-1}\right)$, resembling the spectra of Type IIn SN. These phases in the spectral evolution are shown schematically with arrows. The change to a narrow-line-dominated spectrum is coincident with the very rapid brightening observed in the light curve.

(A color version of this figure is available in the online journal.)

the bolometric correction to convert the I-band luminosities to bolometric luminosities. We use the longer wavelength coverage (from near-UV to $V$ band) of the Swift observations presented in Margutti et al. (2012a) and fit a blackbody to the extinction-corrected fluxes, obtaining the luminosity ratio $L_{\mathrm{bol}} / L_{I} \simeq 13, T_{\mathrm{eff}} \simeq 19200 \mathrm{~K}$, and $R_{\mathrm{bb}} \simeq 2.6 \times 10^{14} \mathrm{~cm}$ on September 26.23. We integrate the $I$-band luminosities estimated from the extinction-corrected magnitudes. Assuming a constant bolometric correction during the 16-day observing period, we obtain a total (optical) radiated energy of $2.7 \times 10^{49} \mathrm{erg}$ and a luminosity of $2 \times 10^{43} \mathrm{erg} \mathrm{s}^{-1}$ as of October 9 . This is already comparable to the estimated radiated energy of the Type IIn SN 2011 ht during its $\sim 100$ day plateau phase (Roming et al. 2012; Mauerhan et al. 2012b).

The evolution of SN 2009ip since the discovery of its latest recorded eruption on 2012 July 24 (Drake et al. 2012) is quite remarkable (Figure 3; see also M12a and P12), as it is unlike any other Type IIn SN studied to date. The absolute magnitude was approximately constant at $\sim-14.5$ ( $V$ to $I$ bands) for a period of $\sim 50$ days after July 24 , and even faded to $\sim-13.5$ (at least between September 18 and September 22.5; Martin et al. 2012; Margutti et al. 2012b) before the rapid brightening that (given our observations) most likely started between September 23.7 and 24.5. If we assume the maximum bolometric correction estimated above as an upper limit and a plateau phase at $M_{I}=-14.5$ for 50 days, we constrain the total radiated energy during this period to be $\lesssim 5 \times 10^{48}$ erg. However, we should 
note that the brightening could have started earlier than July 24, in which case this could be a lower limit in the total radiated energy during this phase.

The spectroscopic evolution of SN 2009ip since its latest recorded eruption has also been unique. Foley et al. (2012) reported an optical spectrum obtained on August 26, which showed a blue continuum with narrow $\left(\sim 700 \mathrm{~km} \mathrm{~s}^{-1}\right)$ emission lines of $\mathrm{H}$ and $\mathrm{He} \mathrm{I}$, similar to the spectra obtained during the 2009 eruption (Smith et al. 2010; Foley et al. 2012). Vinko et al. (2012) reported that an independent spectrum obtained on August 26 also showed broad P-Cygni features with very fast velocities of $\sim 10,000 \mathrm{~km} \mathrm{~s}^{-1}$, consistent with SN shock speeds. These broad features seem to have persisted, and even strengthened, until (at least) September 23 (M12a). However, the high-velocity features and P-Cygni profiles had mostly gone away by September 26-27, leaving behind strong, narrow $\left(\sim 1000 \mathrm{~km} \mathrm{~s}^{-1}\right)$ emission features characteristic of many Type IIn SN spectra (M12a; Burgasser et al. 2012; Vinko et al. 2012; Gall et al. 2012; P12).

As shown in Figure 3, the transition in spectroscopic properties is coincident with the very rapid brightening observed in the optical light curves. This most likely marks the start of a strong interaction between the fast SN shock and a dense circumstellar medium (CSM) environment likely formed from material ejected in the previous LBV outbursts, as proposed by M12a. The timing of the rapid optical brightening is also coincident with the detection of X-ray emission from SN 2009ip (Campana \& Margutti 2012), which is consistent with this picture. Another possible signature of a strong ejecta-CSM interaction is the decline of $\sim 1$ mag observed right before the spectral changes were seen and the light curve started to rise rapidly. Moriya \& Maeda (2012) propose that this is an unavoidable consequence of a fast SN shock breaking out of a dense CSM.

Figure 4 shows a zoom-in view of the evolution of the optical luminosity of SN 2009ip after September 23.5, which is approximately when it started to transition to a Type IIndominated spectrum. For comparison, we also show the optical luminosity evolution of three Type IIn SNe from the literature that have relatively well-sampled light curves before and after peak brightness. This sample includes the very luminous SN 2003ma (Rest et al. 2011) and SN 2006gy (Smith et al. 2007), and the relatively low luminosity SN 2011ht (Roming et al. 2012; Mauerhan et al. 2012b; but, see Humphreys et al. 2012).

The very early rapid optical brightening of the Type IIn SN 2009ip is consistent with $L \propto t^{2}$ during a short two-day phase, although this naturally depends on the $t_{0}$ used. This is the evolution expected for a homologously expanding (optically thick) blackbody photosphere at constant expansion velocity and effective temperature. The luminous Type IIn SN 2003ma and SN 2006gy show relatively similar evolution in their optical luminosity at early times before peak. In fact, Smith \& McCray (2007) modeled the early rise of SN 2006gy with this simple model. SN 2011ht rises at a slower rate in the optical, although it was observed to rise much faster in the UV range (Roming et al. 2012). Given the simplified assumptions that go into the $L \propto t^{2}$ scaling, we plan to explore more realistic models (e.g., Moriya et al. 2013) to fit the early-time light curve of SN 2009ip in a future publication.

At later times, close to peak brightness, the optical luminosity and light curve shape of SN 2009ip become more similar to SN 2011 ht than SN 2003ma or SN 2006gy. Mauerhan et al. (2012b) proposed that SN $2011 \mathrm{ht}$ belongs to a growing sub-class of Type IIn SN (Type IIn-P; including also SN 2009kn (Kankare

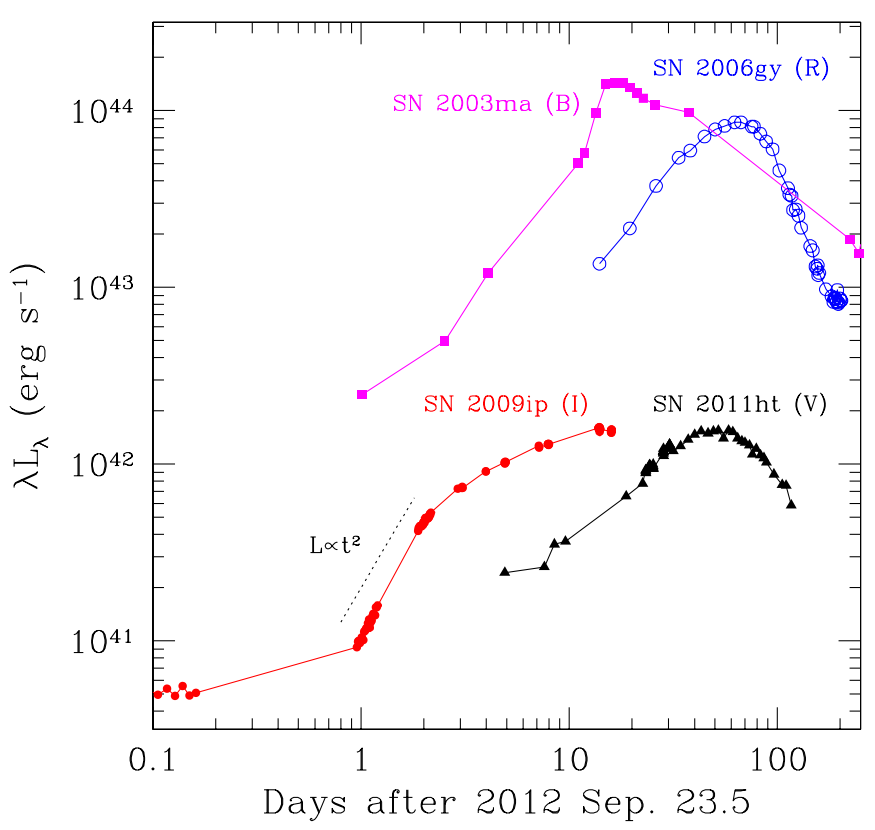

Figure 4. Early rise in optical luminosity of the Type IIn SN 2009ip (filled circles) since 2012 September 23.5. We also show for comparison the luminosity evolution in the rest-frame optical of other Type IIn SN (filters used indicated in parenthesis) with good light-curve coverage before and after peak brightness. These include SN 2003ma (filled triangles; Rest et al. 2011), SN 2006gy (open circles; Smith et al. 2007), and SN 2011ht (filled squares; Roming et al. 2012). We have used the distances, extinctions, and $K$-corrections provided in each paper to derive the luminosities. The time in these cases is estimated with respect to an approximate explosion date (JD = 2,452,971.0 for SN 2003ma and JD $=2,453,967.0$ for SN 2006gy) or with respect to the discovery date ( $\mathrm{JD}=2,455,833.7$ for SN 2011ht). The dotted line shows the luminosity scaling $L \propto t^{2}$.

(A color version of this figure is available in the online journal.)

et al. 2012) and SN 1994W (Sollerman et al. 1998)) with long-lasting post-peak plateaus of $\sim 100$ days ( $M \sim-18 \mathrm{mag}$ ), and faint late-time decay slopes consistent with low ${ }^{56} \mathrm{Ni}$ yield production $\left(\sim 0.01 M_{\odot}\right)$. Perhaps we are seeing something similar in the case of SN 2009ip (but, see P12), although there are important differences in spectroscopic properties compared to the small group of Type IIn-P SN (e.g., SN 2009ip shows significantly hotter continuum and the narrow lines do not show P-Cygni profiles). An interesting related issue that comes out from the recent observations of SN 2009ip before the rapid brightening and fast transition to narrow-line-dominated spectrum, is whether surveys might be missing a relatively low-luminosity phase (at least compared to the main peak) in which the broad SN shock velocities are revealed. Given the typical depth of transient surveys, this is certainly possible (e.g., see Figure 17 in Kiewe et al. 2012). In fact, Smith et al. (2011b) proposed such an origin for the $\sim 100$ day plateau at $M \sim-16$ mag observed before the main peak in the light curve of SN $1961 \mathrm{~V}$.

\section{CONCLUSIONS}

We have presented high-cadence optical photometric monitoring of the remarkable, young Type IIn supernova SN 2009ip using data obtained between 2012 September 23 and October 9 with 0.3-0.4 $\mathrm{m}$ aperture telescopes from the Coral Towers Observatory (Cairns, Australia). Using difference imaging analysis, we obtain precise $R$ - and $I$-band light curves and are able to resolve well-defined brightening phases. In particular, we see a very rapid brightening early on $(0.5 \mathrm{mag}$ in $6 \mathrm{hr})$ that quickly turns over after a couple of days. The SN brightened between 
absolute magnitude $M_{I}=-14.2$ and $M_{I}=-17.9$ in 16 days. The changes that we observe in the light curves are correlated with the reported spectroscopic changes and the X-ray detection and most likely mark the start of a strong interaction between the fast SN ejecta and the dense CSM formed from material that was ejected in the pre-SN LBV eruptions observed since 2009. Our study highlights the importance of organized, highcadence observations of transients by amateur astronomers with small-aperture telescopes.

We thank S. de Mink, O. Graur, J. Mauerhan, R. Quimby, and A. Rest for stimulating discussions about SN 2009ip, and L. Watson for detailed comments on an earlier version of this manuscript. We thank the anonymous referee for detailed comments. J.L.P. acknowledges support from a Carnegie-Princeton Fellowship.

\section{REFERENCES}

Alard, C. 2000, A\&AS, 144, 363

Arnett, W. D., \& Meakin, C. 2011, ApJ, 733, 78

Brimacombe, J. 2012, ATel, 4423, 1

Burgasser, A., Nicholls, C., \& Aberasturi, M. 2012, ATel, 4431, 1

Campana, S., \& Margutti, R. 2012, ATel, 4499, 1

Chevalier, R. A. 2012, ApJL, 752, 2

Drake, A. J., Howerton, S., McNaught, R., et al. 2012, ATel, 4334, 1

Drake, A. J., Prieto, J. L., Djorgovski, S. G., et al. 2010, ATel, 2897, 1

Foley, R. J., Berger, E., Fox, O., et al. 2011, ApJ, 732, 32

Foley, R. J., Berger, E., Roederer, I. U., \& Chomiuk, L. 2012, ATel, 4338, 1

Gall, C., Hjorth, J., \& Leloudas, G. 2012, ATel, 4454, 1

Gal-Yam, A., \& Leonard, D. C. 2009, Natur, 458, 865

Gal-Yam, A., Leonard, D. C., Fox, D. B., et al. 2007, ApJ, 656, 372

Hartman, J. D., Bakos, G., Stanek, K. Z., \& Noyes, R. W. 2004, AJ, 128,1761
Humphreys, R. M., \& Davidson, K. 1994, PASP, 106, 1025

Humphreys, R. M., Davidson, K., Jones, T. J., et al. 2012, ApJ, 760, 93

Kankare, E., Ergon, M., Bufano, F., et al. 2012, MNRAS, 424, 855

Kiewe, M., Gal-Yam, A., Arcavi, I., et al. 2012, ApJ, 744, 10

Kochanek, C. S., Beacom, J. F., Kistler, M. D., et al. 2008, ApJ, 684, 1336

Kochanek, C. S., Szczygiel, D. M., \& Stanek, K. Z. 2011, ApJ, 737, 76

Kochanek, C. S., Szczygiel, D. M., \& Stanek, K. Z. 2012, ApJ, 758, 142

Langer, N. 2012, ARA\&A, 50, 107

Li, W., Leaman, J., Chornock, R., et al. 2011, MNRAS, 412, 1441

Margutti, R., Soderberg, A., Chornock, R., \& Foley, R. 2012a, ATel, 4425, 1

Margutti, R., Soderberg, A., \& Milisavljevic, D. 2012b, ATel, 4414, 1

Martin, J. C., O'Brien, J., \& Hubbell-Thomas, J. 2012, ATel, 4416, 1

Mauerhan, J. C., Smith, N., Filippenko, A., et al. 2012a, arXiv:1209.6320 (M12a)

Mauerhan, J. C., Smith, N., Silverman, J. M., et al. 2012b, arXiv:1209.0821

Maza, J., Hamuy, M., Antezana, R., et al. 2009, CBET, 1928, 1

Moriya, T. J., Blinnikov, S. I., Tominaga, N., et al. 2013, MNRAS, 428, 1020

Moriya, T. J., \& Maeda, K. 2012, ApJL, 756, 22

Pastorello, A., Cappellaro, E., Inserra, C., et al. 2012, arXiv:1210.3568 (P12)

Pastorello, A., Smartt, S. J., Mattila, S., et al. 2007, Natur, 447, 829

Prieto, J. L., Brimacombe, J., \& Drake, A. J. 2012, ATel, 4439, 1

Quataert, E., \& Shiode, J. 2012, MNRAS, 423, L92

Rest, A., Foley, R. J., Gezari, S., et al. 2011, ApJ, 729, 88

Roming, P. W. A., Pritchard, T. A., Prieto, J. L., et al. 2012, ApJ, 751, 92

Smartt, S. J. 2009, ARA\&A, 47, 63

Smith, N., Li, W., Foley, R. J., et al. 2007, ApJ, 666, 1116

Smith, N., Li, W., Miller, A. A., et al. 2011a, ApJ, 732, 63

Smith, N., Li, W., Silverman, J. M., Ganeshalingam, M., \& Filippenko, A. V. 2011b, MNRAS, 415, 773

Smith, N., \& Mauerhan, J. 2012a, ATel, 4412, 1

Smith, N., \& Mauerhan, J. 2012b, ATel, 4427, 1

Smith, N., \& McCray, R. 2007, ApJL, 671, 17

Smith, N., Miller, A., Li, W., et al. 2010, AJ, 139, 1451

Sollerman, J., Cumming, R. J., \& Lundqvist, P. 1998, ApJ, 493, 933

Stetson, P. B. 1992, ASPC, 25, 297

Stritzinger, M., Taddia, F., Fransson, C., et al. 2012, ApJ, 756, 173

Vinko, J., Wheeler, J. C., Milisavljevic, D., et al. 2012, ATel, 4435, 1

Woosley, S. E., Blinnikov, S., \& Heger, A. 2007, Natur, 450, 390 